\title{
Water Droplet Motion on an Inclining Surface
}

\author{
Thomas Maurer, Axel Mebus, Uwe Janoske \\ University of Wuppertal, Chair of Fluid Mechanics \\ Gaußstraße 20, 42097 Wuppertal, Germany \\ maurer@uni-wuppertal.de; janoske@uni-wuppertal.de
}

\begin{abstract}
Droplet motion induced by external forces such as mechanical vibrations, shear flows or gravitational forces has a major importance in many industrial applications. The present study introduces a setup for a tilting plane experiment, which enables investigations of the droplet behaviour due to the balance between surface tension and gravitational force. Furthermore, droplet motion measurements on a tilting acrylic glass surface are presented. The main aspect of this study is the influence of the droplet volume and the angular velocity of the inclining plane on the droplet detachment. To quantify this influence, different moving regimes are detected and specified. Further, flow maps due to the rotational velocity are obtained. The results show that the inclination angle needed to initiate drop motions decreases when the droplet volume increases. In addition to that, the droplet motion is initiated at a smaller inclination angle if the angular velocity decreases. This behaviour has no influence when rotational velocity increases above a certain threshold. The study concludes that the detachment of water droplets on a tilting acrylic surface can be forced and amplified if either the droplet volume is increased or the angular velocity of the surface is decreased.
\end{abstract}

Keywords: contact angle hysteresis, droplet, inclination, gravitation, contact line, wetting, detachment

\section{Introduction}

The field of droplet movement research has been of great interest in recent years. The correlation between different liquid properties and surface materials due to practical applications such as wetting and dewetting of both, hydrophilic and hydrophobic surfaces, has been investigated in detail. For this purpose extensive analysis of droplets on an inclined or inclining plane were carried out. This is done by measuring contact angles and sliding angles of moving droplets of different sizes on versatile substrates. Table 1 shows an overview of the most recent publications in this field of research.

The first subdivided column of table 1 illustrates the major interests of the listed investigations. Three main effects were explored for this purpose: the influence of the droplet volume on the critical slope angle, the dependency of the angular rotational velocity on droplet motion and the change of droplet contour due to the inclination angle. Das et al. [1] investigated the droplet motion of water on a proton-exchange-membrane fuel-cell gas-diffusion layer. In their experiment, they used a modified automated goniometer for the measurement of contact angles and sliding angles of different water droplet sizes (515 $\mu$ l). The droplets were placed using two injection methods (on the top and through the bottom of the substrate) and two placement methods (placed on a horizontal surface and placed on a pre-tilted surface). The rotary motor operates with a constant speed mostly between $0.5 \%$ and $1 \%$. The authors used the results to calculate the adhesion force between the gas diffusion layer surface and the droplets and came to the conclusion that for droplet volumes larger than $10 \mu 1$ the sliding angles are higher for droplets created on a horizontal surface than for droplets created on a pre-tilted surface. For small drops the sliding angles are almost identical.

The second group of columns illustrates the methods of droplet placement which have been investigated. Droplets can be placed horizontally, vertically, on a fixed pre-tilted surface or on a constant inclining surface. Quéré [2] used a tilted surface with a fixed inclination for his investigations. He examined the case of a small contact angle hysteresis, for which the drops are found to resemble spherical caps, despite the slope. Most of the experiments used an inclining plane apparatus. In order to calculate the adhesion force for drops, Antonini et al. [3] developed an alternative method for the recording and the analysis of the droplet shape on inclining surfaces. This method is based on the usage of multiple profile images of droplets. In the experimental setup images of a droplet on an inclined surface are recorded by a camera, which rotates $180^{\circ}$ around the drop taking photographs at $10^{\circ}$ steps. The images are used to accurately reconstruct the contact line shape and the contact angle distribution along the contact line. This novel method also eliminates the problem of perspective error. 
The columns headed "angular velocity" illustrate the rotational velocity of the tilting plate apparatus experiments. Extrand and Kumagi [4] tilted the surface with an angular speed of 0.7 to $1 \%$. The authors investigated the correlation between the two different contact liquids - water and ethylene glycol - in relation to four polymers and silicon wafers. The setup used four drop volumes between 36 and $116 \mu \mathrm{l}$ for experiments on a rotatable plane apparatus. From the experimental data the authors draw the conclusion that the hysteresis is closely tied to the chemical nature and that the roughness of the surfaces has a negligible influence. Lv et al. [5] applied the highest angular speed with $1.5 \%$ for all but for the present investigation. The authors investigated the correlation between water droplets and pillar-structured hydrophobic surfaces. For measurements with droplet volumes of 5,10 and $15 \mu 1$ they used a commercial contact angle meter. The droplets were quasi-statically placed on the samples by an automatical micro syringe. The researchers observed that the onset of droplet sliding under gravity on inclined pillar-structured hydrophobic surfaces always starts with detachment of the rear contact line. The majority of investigated droplet volumes ranges from 1 to $50 \mu 1$. Most of the investigations use hydrophobic surfaces.

Within the scope of the present article we take a closer look onto the droplet motion behaviour on an inclining hydrophilic acrylic glass substrate with different constant angular velocities. To the best knowledge of the authors, the influence of the angular velocity on the droplet motion has not yet been investigated in detail. The experimental setup for the investigation of a droplet motion on inclining surfaces is presented in the following chapter. We rely on the tilted plane method in order to investigate the movement of drops due to the change of force balance between surface tension force of the droplet and the gravitational force.

\section{Experimental Setup}

The scheme of the apparatus designed and realized within the scope of this study is shown in figure 1 . The experimental setup consists of three main parts: droplet generation and positioning, a tilting plate apparatus and an imaging system.

A micro syringe is used to generate water droplets of different sizes $(5-30 \mu 1)$. The accuracy of the droplet generation is verified by means of an analytical balance. The droplet weight is measured for twenty times for each investigated droplet size. The results show a very good reproducibility of the droplet size with an average standard deviation of $\sigma=0.02 \mu l$. In order to ensure repeatability in placement, the droplet is formed on the tip of the syringe and gently lowered to the acrylic surface, which is placed on the tilting plate.

The tilting apparatus consists of the tilting plate, which is mounted to a tube. The tube is connected to a double stage toothed belt drive with a gear reduction of 9:1. To tilt the acrylic plate with a constant rotational velocity, the gearing mechanism is mechanically powered by a step motor. The step angle is $0.0072^{\circ}$ and the rotational speed is reduced by an internal reduction of 50:1. This setup makes it possible to realize very slow and very high angular velocities. Moreover, the step motor is installed on a vibration absorbing plate, to lower the transfer of vibrations of the step motor to the water droplet. Finally different rotational speeds of the tilting plate $(5,10,20,40,50 \% \mathrm{~s})$ are realized and controlled by means of a monitoring software.

The imaging system involves a high speed camera, a macro objective and a cold light illumination unit. As shown in figure 1, the drop is positioned between the objective of the camera and the light source so that the drop is backlit. A diffuser placed between the light source and the tube of the tilting apparatus ensures uniform illumination.

The apparatus has to be calibrated before the measurements can be conducted. The tilting plate needs to be aligned horizontal. For each droplet size the position of the camera, the zoom level of the objective as well as the focusing onto the droplet have to be varied such that the droplet, while it is tilted, is displayed in the centre of the photographs.

All measurements are conducted at equal ambient conditions, realized by an air conditioning system. In every measurement the tilting plate is inclined from 0 to $90^{\circ}$. We measure six different droplet sizes $(5,10,15,20,25,30 \mu \mathrm{l})$ and every droplet size with four different constant rotational speeds $(10,20,40,50 \%$ s). For the analysis it is important that an adequate amount of photographs is taken. For this reason, the frame rate for the rotational speeds of 10 and $20 \%$ is set to $60 \mathrm{fps}$ and for 40 and $50 \%$ s the framerate is set to $120 \mathrm{fps}$.

This experimental setup allows the investigation of a droplet on an inclined surface with a wide range of different angular velocities. Figure 2 shows the final experimental setup. 
Table 1: Overview of the recent literature on droplet motion on a tilted or tilting substrate.

\begin{tabular}{|c|c|c|c|c|c|c|c|c|c|c|c|c|c|c|c|c|}
\hline \multirow[b]{2}{*}{ year } & \multirow[b]{2}{*}{ authors } & \multicolumn{3}{|c|}{ interest } & \multicolumn{4}{|c|}{ droplet position } & \multicolumn{4}{|c|}{ angular velocity } & \multicolumn{4}{|c|}{ droplet volume } \\
\hline & & 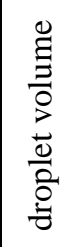 & 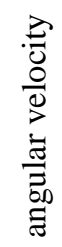 & 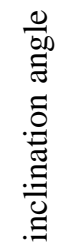 & 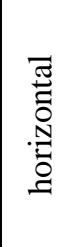 & 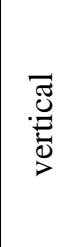 & $\begin{array}{l}\stackrel{\Xi}{\Xi} \\
\stackrel{\Xi}{Z} \\
\stackrel{\Xi}{\Xi} \\
. \Xi \\
. \Xi\end{array}$ & $\begin{array}{l}\stackrel{0}{\Xi} \\
0 \\
000 \\
. \Xi \\
. \Xi \\
. \Xi\end{array}$ & $\begin{array}{l}\infty \\
0 \\
0 \\
1 \\
1 \\
0\end{array}$ & 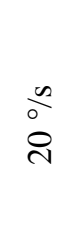 & 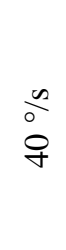 & $\begin{array}{l}\frac{\infty}{0} \\
0 \\
i\end{array}$ & $\begin{array}{c}3 \\
0 \\
1 \\
1\end{array}$ & $\begin{array}{l}\Xi \\
\stackrel{N}{0} \\
1 \\
=\end{array}$ & $\begin{array}{l}\bar{z} \\
0 \\
i \\
i \\
\bar{N}\end{array}$ & $\begin{array}{l}\overline{5} \\
\circ \\
\frac{1}{1} \\
\bar{n}\end{array}$ \\
\hline 1994 & Extrand, Kumagai [6] & & & & & & & - & & & & & & & - & - \\
\hline 1994 & Shiiba et al. [7] & & & & & & - & & & & & & & & & \\
\hline 1997 & Extrand, Kumagai [4] & & & & & & & $\bullet$ & $\bullet$ & & & & & & - & - \\
\hline 1997 & Quéré [2] & & & & $\bullet$ & $\bullet$ & $\bullet$ & & & & & & & & & \\
\hline 1999 & Miwa et al. [8] & $\bullet$ & & & & & $\bullet$ & & & & & & $\bullet$ & $\bullet$ & • & \\
\hline 1999 & Erbil et al. [9] & & & & & & & - & & & & & $\bullet$ & & & \\
\hline 1999 & Richard et al. [10] & & & & & & & $\bullet$ & & & & & & & & \\
\hline 2002 & Yoshimitsu et al. [11] & $\bullet$ & & & & & & $\bullet$ & & & & & $\bullet$ & $\bullet$ & $\bullet$ & \\
\hline 2003 & Marmur [12] & & & & & & & & & & & & & & & \\
\hline 2003 & ElSherbini et al. [13] & & & & & & & - & & & & & & & & \\
\hline 2004 & Ha et al. [14] & $\bullet$ & & & & & & - & $\bullet$ & & & & $\bullet$ & & & \\
\hline 2004 & Shirtcliffe et al. [15] & & & & & & & - & & & & & & $\bullet$ & & \\
\hline 2004 & Krasovitski [16] & & & & & & & & & & & & & & & \\
\hline 2005 & Martines et al. [17] & $\bullet$ & & & $\bullet$ & & & - & & & & & $\bullet$ & $\bullet$ & $\bullet$ & \\
\hline 2006 & Sakai et al. [18] & & & & & & $\bullet$ & & & & & & & & $\bullet$ & \\
\hline 2007 & Cortese et al. [19] & & & & & & & - & & & & & $\bullet$ & & & \\
\hline 2008 & Pierce et al. [20] & $\bullet$ & & & $\bullet$ & & - & - & $\bullet$ & & & & & - & $\bullet$ & $\bullet$ \\
\hline 2008 & Hashimoto et al. [21] & & & & & & - & & & & & & $\bullet$ & $\bullet$ & - & \\
\hline 2008 & Xiu et al. [23] & & & & $\bullet$ & & & & & & & & $\bullet$ & & & \\
\hline 2009 & Antonini, et al. [3] & & & & & & & - & & & & & & & & \\
\hline 2010 & Lv et al. [5] & $\bullet$ & & & & & & - & $\bullet$ & & & & $\bullet$ & - & & \\
\hline 2010 & Spori et al. [24] & & & & & $\bullet$ & & $\bullet$ & & & & & $\bullet$ & & & \\
\hline 2011 & Ruiz-Cabello [4] & & & $\bullet$ & & & & $\bullet$ & & & & & & & & $\bullet$ \\
\hline 2011 & Mertaniemi et al. [25] & & & & & & $\bullet$ & & & & & & $\bullet$ & & & \\
\hline 2012 & Das et al. [1] & - & & & & & & - & $\bullet$ & & & & $\bullet$ & - & & \\
\hline 2016 & Present study & $\bullet$ & • & $\bullet$ & & & & $\bullet$ & $\bullet$ & $\bullet$ & $\bullet$ & $\bullet$ & $\bullet$ & $\bullet$ & $\bullet$ & \\
\hline
\end{tabular}




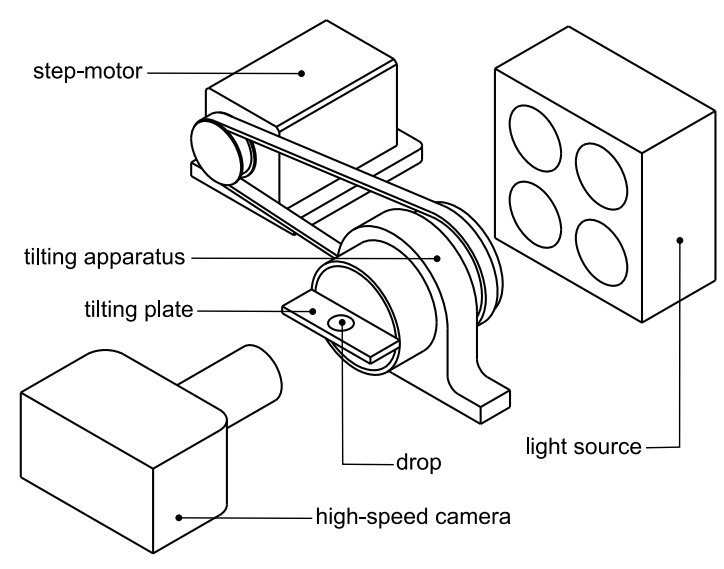

Fig. 1: Schematic experimental setup.

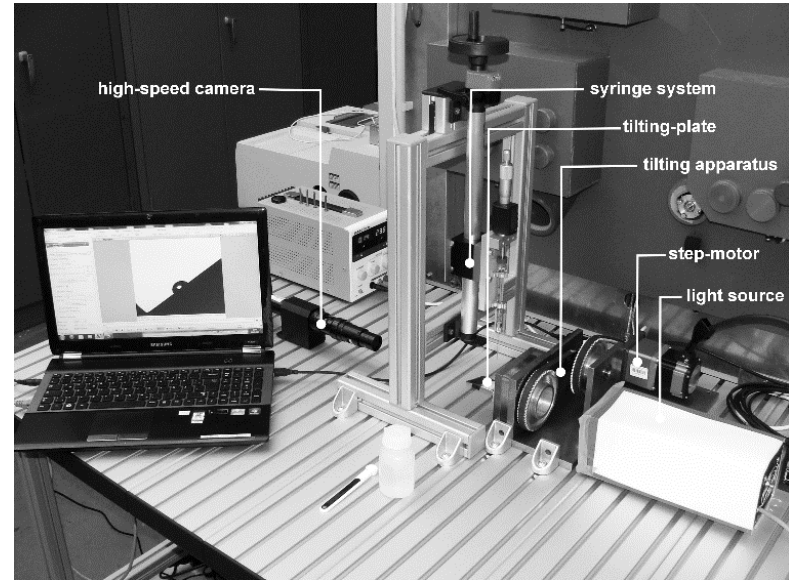

Fig. 2: Experimental realization.

\section{Results}

We present the results of our study in terms of the critical tilting angle $\alpha_{\text {crit. }}$ This angle describes the instant when the droplet motion is initialized due to the force imbalance between the surface tension force and the gravitational force. In all subsequent experiments the droplet position is varied from the horizontal to the vertical position using different rotational speeds.

\subsection{Length development of the droplet}

Figure 3 shows that the length development of a $20 \mu 1$ droplet on an inclining plane can be characterized into four different moving regimes:

state 0: static droplet state,

state 1: advancing motion state,

state 2: receding motion state,

state 3: droplet detachment.

For very small gravitational changes the initial droplet contour and therefore the static state is observed (state 0). In the next regime, the receding end induces a reluctant behaviour of the droplet, whereas the enhancement of the inclination angle leads to a motion of the advancing part. This results in an increasing droplet length (state 1). This behaviour is observed as long as the gravitational force of the droplet and the force at the leading end is smaller than the retaining force at the rear of the droplet. At the highest point of state $1\left(\sim 66^{\circ}\right)$ a balance is reached between the gravitational force and the surface force of the droplet. Afterwards the droplet behaviour reverses. The receding end starts to move and the advancing end holds of the contact line. As a consequence, the droplet length is reduced (state 2). Before an explicit droplet movement (state 3) can be detected, the droplet passes again through the first and the second regime (70-78 ). Afterwards the droplet accelerates downwards the inclining plane and the droplet length increases. This droplet motion (state line 1-2-1-2-3) is observed for droplets of a volume between 20-30 $\mu$ l. For smaller droplets the droplet detachment cannot be detected due to the lack of droplet mass.

Figure 4 shows the length development of a $30 \mu \mathrm{l}$ droplet on an inclining plane with an angular velocity of $20^{\circ} / \mathrm{s}$. Unlike to droplets with a volume between 20-30 $\mu 1$, the state line simplifies to 1-2-3. Moreover, the beginning of every state occurs earlier due to a higher droplet mass. The complete droplet movement (state 3) starts at $55^{\circ}$ and not at $75^{\circ}$ as in figure 3 . Hence, increasing the droplet volume leads to an earlier droplet detachment. Figure 5 shows the different regimes for a $30 \mu \mathrm{l}$ droplet. The first two images show the static state where the droplet contour does not change much. Compared to that at an inclination of $40^{\circ}$ the droplet length is increased. For a slope of $55^{\circ}$ the droplet detachment is initialized and the droplet motion is visualized for a tilting angle of $70^{\circ}$. 


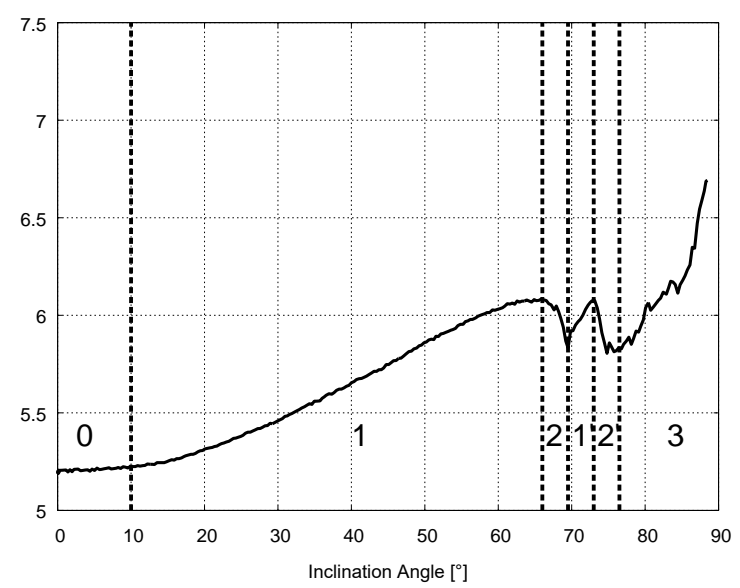

Fig. 3: Length development of a $20 \mu 1$ droplet.

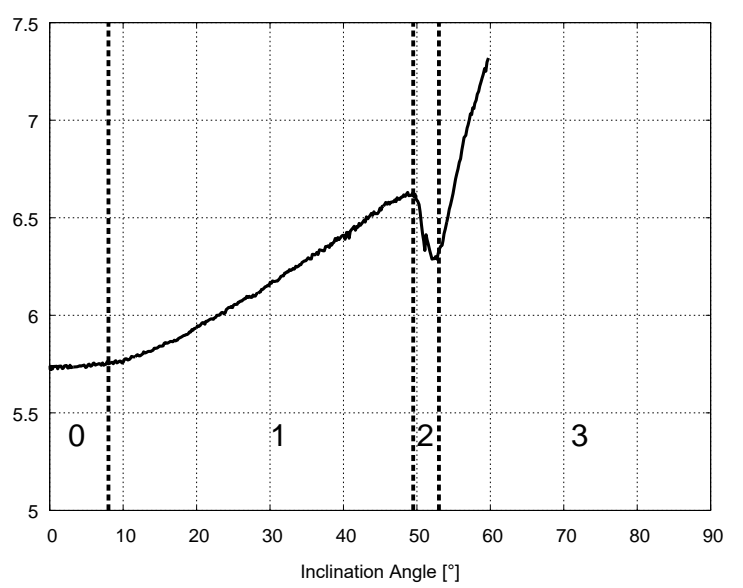

Fig. 4: Length development of a $30 \mu 1$ droplet.

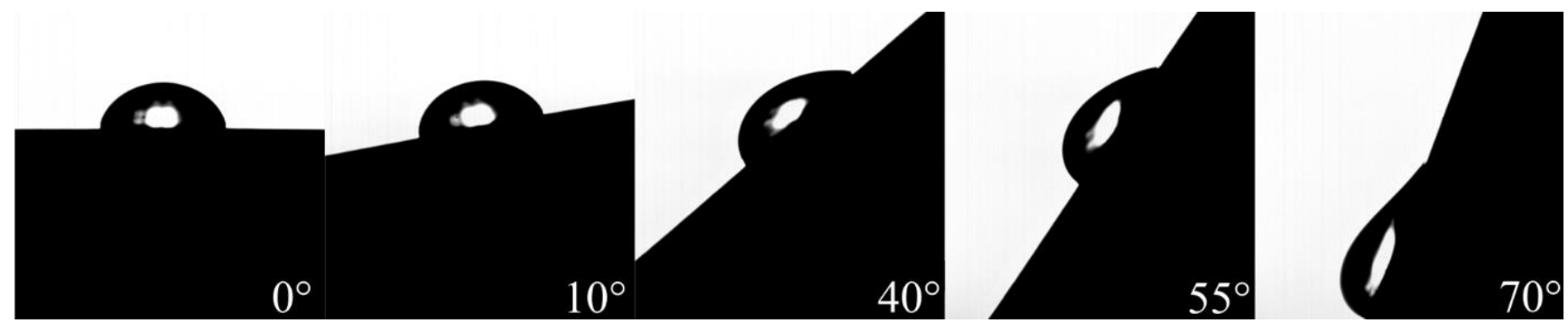

Fig. 5: Different droplet regimes for a $30 \mu 1$ droplet.

\subsection{Flow map of the droplet}

Figures 6 and 7 show the influence of the rotational speed on the regime transition. Droplets with a volume smaller than $20 \mu \mathrm{l}$ do not cross the transition from state 2 to state 3 by tilting the angle with $10 \%$ s (figure 6 ). Thus, a droplet motion is not detected here. For droplets smaller than $15 \mu \mathrm{l}$ only the static and advancing acceleration regime exists. Finally, increasing the droplet volume to $30 \mu 1$ leads to an earlier transition from state 1 to state 3 .

Comparing these results with those presented in figure 7 it becomes evident, that all transition areas are shifted to a higher inclination angle by increasing the angular velocity to $20 \%$. This is due to the fact that the droplet has more time to develop a stable position when the influence of the gravitational force is not increased too fast. For example, the droplet detachment for a rotational speed of $20^{\circ}$ /s occurs for a $20 \mu 1$ droplet at $78^{\circ}$ instead of $66^{\circ}$ for $10^{\circ} \%$ s.

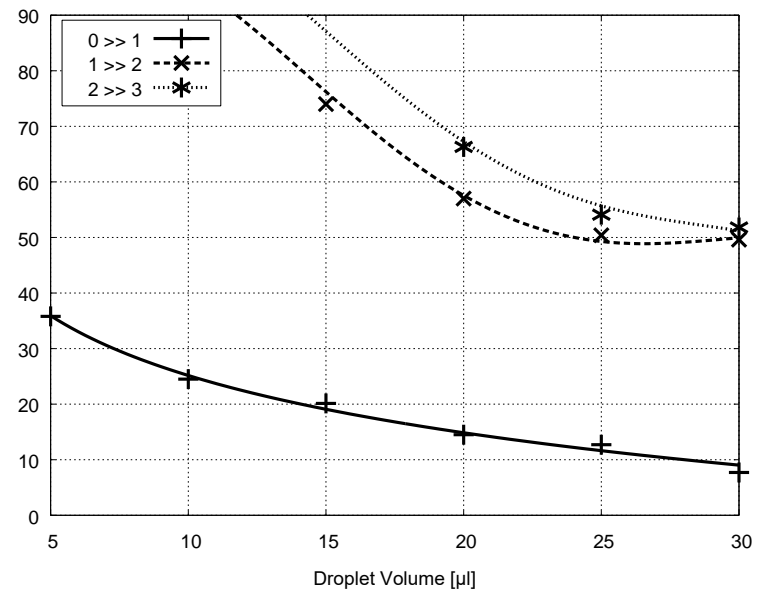

Fig. 6: Flow map for an angular velocity of $10 \%$ s.

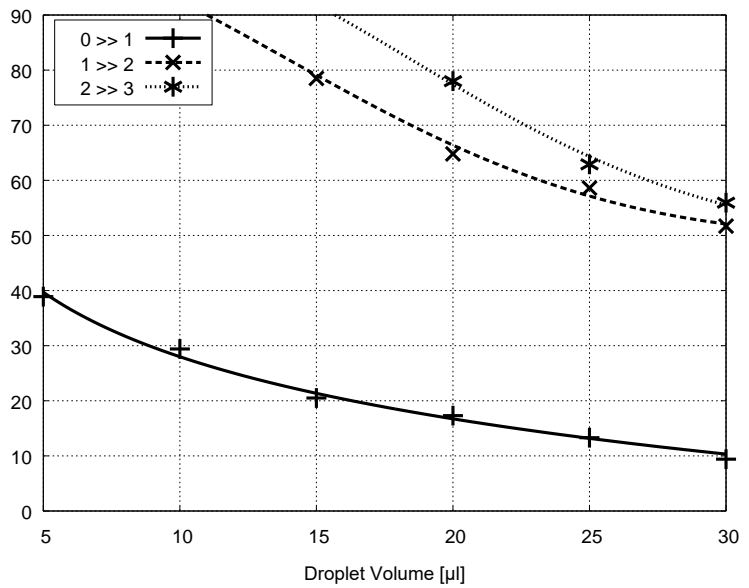

Fig. 7: Flow map for an angular velocity of $20 \%$ s. 
Increasing the rotational velocity to $40 \% \mathrm{~s}$ and $50 \%$ (figure 8 and 9) confirms the observed results that the droplet motion is again induced at a smaller inclination angles. The moving maps between $40 \% \mathrm{~s}$ and $50 \%$ do not differ significantly. For a $30 \mu \mathrm{l}$ droplet the transition regions occur at approximately $17^{\circ}, 53^{\circ}$ and $61^{\circ}$ for both rotational velocities. Hence, for angular velocities above a certain threshold, the droplet experiences a similar moving behaviour.

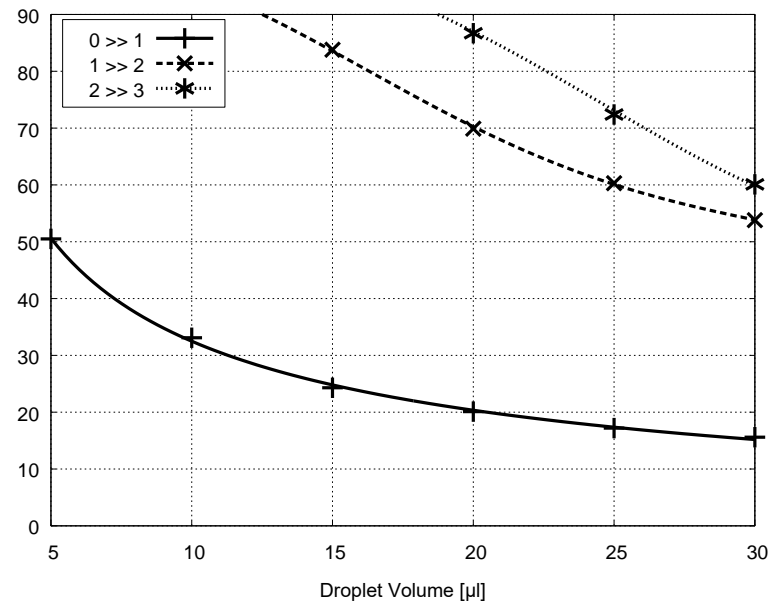

Fig. 8: Flow map for an angular velocity of $40 \%$ s.

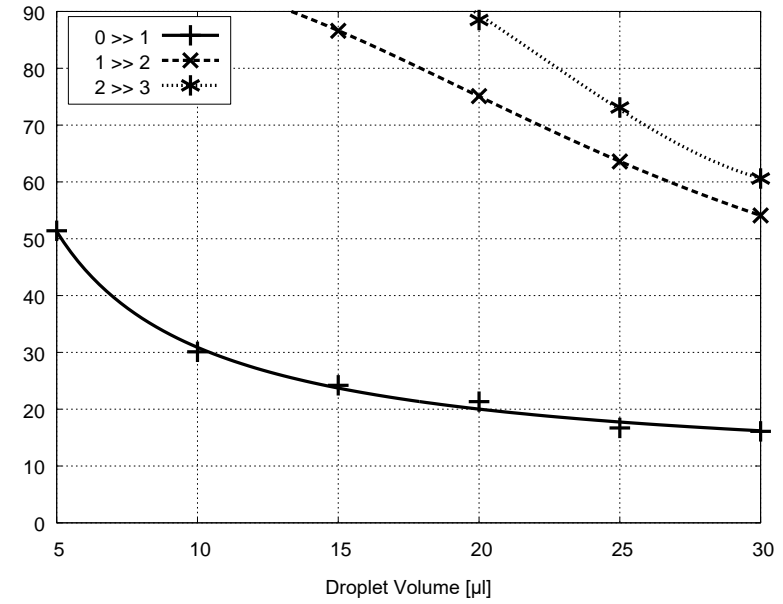

Fig. 9: Flow map for an angular velocity of $50 \%$ s.

\section{Conclusion}

This study presents an experimental setup for the investigation of water droplet motion caused by the balance between the surface tension force and the gravitational force. Furthermore, results of droplet motion measurements on an inclining acrylic glass surfaces are documented. The inclination of the surface is realized by means of a step motor droplet motions are visualized by a diffused back light in combination with a high-speed camera. The post-processing of the achieved images uses an in-house code which is based on a grey-scale analysis of the images. The study aims at investigating the moving behaviour of the droplet and determining the influence of the rotational speed of the surface on the droplet detachment point.

Inclination measurements at a constant rotational velocity but with different droplet volumes show that droplets smaller than $30 \mu \mathrm{l}$ have a different detachment scheme than droplets with volume larger than $30 \mu 1$. Small droplets $(\leq 30 \mu \mathrm{l})$ iteratively traverse different moving regimes. At the beginning a motion at the advancing part is detected which is followed by a motion at the receding part. Before the droplet detaches both regimes occur again. Larger droplets (volume $\geq 30 \mu \mathrm{l}$ ) show a simpler moving scheme. The droplet length increases due to the movement of the advancing area and it decreases at the receding part. For larger droplet volumes the transition from the static to dynamic state occurs at smaller inclination angle.

Furthermore, the results clearly indicate that droplets smaller than $20 \mu 1$ do not detach from the surface. Above this droplet volume an enhancement of the droplet volume leads to an earlier detachment. Decreasing the rotational speed results also in an earlier droplet motion. The influence of the angular velocity reduces above a magnitude of $40 \%$ s.

The experiments show that the influence of angular velocity on the initial droplet motion increases with higher droplet volume. We therefore conclude that the detachment of water droplets on acrylic glass surfaces does increase significantly when the droplet volume increases and the rotational velocity decreases.

\section{References}

[1] P. K. Das, A. Grippin, A. Kwong, and A. Z. Weber, "Liquid-Water-Droplet Adhesion-Force Measurements on Fresh and Aged Fuel-Cell Gas-Diffusion Layers," Journal of The Electrochemical Society, vol. 159, no. 5, pp. B489, 2012.

[2] D. Quéré, M.-J. Azzopardi, and L. Delattre, "Drops at Rest on a Tilted Plane," Langmuir, vol. 14, no. 8, pp. 2213-2216, 1998.

[3] C. Antonini, F. J. Carmona, E. Pierce, M. Marengo, and A. Amirfazli, "General Methodology for Evaluating the Adhesion Force of Drops and Bubbles on Solid Surfaces,” Langmuir, vol. 25, no. 11, pp. 6143-6154, 2009. 
[4] C.W. Extrand and Y. Kumagai, “An Experimental Study of Contact Angle Hysteresis," Journal of Colloid and Interface Science, vol. 191, no. 2, pp. 378-383, 1997.

[5] C. Lv, C. Yang, P. Hao, F. He, and Q. Zheng, "Sliding of Water Droplets on Microstructured Hydrophobic Surfaces," Langmuir, vol. 26, no. 11, pp. 8704-8708, 2010.

[6] C. W. Extrand and Y. Kumagai, "Liquid Drops on an Inclined Plane: The Relation between Contact Angles, Drop Shape, and Retentive Force," Journal of Colloid and Interface Science, vol. 170, no. 2, pp. 515-521, 1995.

[7] J. Fukai, Y. Shiiba, T. Yamamoto, O. Miyatake, D. Poulikakos, C. M. Megaridis, and Z. Zhao, "Wetting effects on the spreading of a liquid droplet colliding with a flat surface: Experiment and modeling," Physics of Fluids, vol. 7, no. 2, p. 236, 1995.

[8] M. Miwa, A. Nakajima, A. Fujishima, K. Hashimoto, and T. Watanabe, "Effects of the Surface Roughness on Sliding Angles of Water Droplets on Superhydrophobic Surfaces," Langmuir, vol. 16, no. 13, pp. 5754-5760, 2000.

[9] H. Yildirim Erbil, G. McHale, S. M. Rowan, and M. I. Newton, "Determination of the Receding Contact Angle of Sessile Drops on Polymer Surfaces by Evaporation," Langmuir, vol. 15, no. 21, pp. 7378-7385, 1999.

[10] D. Richard and D. Quéré, "Viscous drops rolling on a tilted non-wettable solid," Europhysics Letters (EPL), vol. 48, no. 3, pp. 286-291, 1999.

[11] Z. Yoshimitsu, A. Nakajima, T. Watanabe, and K. Hashimoto, "Effects of Surface Structure on the Hydrophobicity and Sliding Behavior of Water Droplets," Langmuir, vol. 18, no. 15, pp. 5818-5822, 2002.

[12] A. Marmur, "The Lotus Effect: Superhydrophobicity and Metastability," Langmuir, vol. 20, no. 9, pp. 3517-3519, 2004.

[13] A. I. ElSherbini and A. M. Jacobi, "Liquid drops on vertical and inclined surfaces," Journal of Colloid and Interface Science, vol. 273, no. 2, pp. 556-565, 2004.

[14] J.-W. Ha, I. J. Park, and S.-B. Lee, "Hydrophobicity and Sliding Behavior of Liquid Droplets on the Fluorinated Latex Films," Macromolecules, vol. 38, no. 3, pp. 736-744, 2005.

[15] N. J. Shirtcliffe, G. McHale, M. I. Newton, and C. C. Perry, "Wetting and Wetting Transitions on Copper-Based SuperHydrophobic Surfaces," Langmuir, vol. 21, no. 3, pp. 937-943, 2005.

[16] B. Krasovitski and A. Marmur, "Drops Down the Hill: Theoretical Study of Limiting Contact Angles and the Hysteresis Range on a Tilted Plate," Langmuir, vol. 21, no. 9, pp. 3881-3885, 2005.

[17] E. Martines, K. Seunarine, H. Morgan, N. Gadegaard, C. D. W. Wilkinson, and M. O. Riehle, "Superhydrophobicity and Superhydrophilicity of Regular Nanopatterns," Nano Letters, vol. 5, no. 10, pp. 2097-2103, 2005.

[18] M. Sakai, J.-H. Song, N. Yoshida, S. Suzuki, Y. Kameshima, and A. Nakajima, "Direct Observation of Internal Fluidity in a Water Droplet during Sliding on Hydrophobic Surfaces," Langmuir, vol. 22, no. 11, pp. 4906-4909, 2006.

[19] B. Cortese, S. D'Amone, M. Manca, I. Viola, R. Cingolani, and G. Gigli, "Superhydrophobicity Due to the Hierarchical Scale Roughness of PDMS Surfaces,” Langmuir, vol. 24, no. 6, pp. 2712-2718, 2008.

[20] E. Pierce, F.J. Carmona, and A. Amirfazli, "Understanding of sliding and contact angle results in tilted plate experiments," Colloids and Surfaces A: Physicochemical and Engineering Aspects, vol. 323, no. 1-3, pp. 73-82, 2008.

[21] A. Hashimoto, M. Sakai, J.-H. Song, N. Yoshida, S. Suzuki, Y. Kameshima, and A. Nakajima, "Direct Observation of Water Droplet Motion on a Hydrophobic Self-assembled Monolayer Surface under Airflow," Journal of The Surface Finishing Society of Japan, vol. 59, no. 12, pp. 907-912, 2008.

[22] Y. Xiu, L. Zhu, D. W. Hess, and C. P. Wong, "Relationship between Work of Adhesion and Contact Angle Hysteresis on Superhydrophobic Surfaces," J. Phys. Chem. C, vol. 112, no. 30, pp. 11403-11407, 2008.

[23] D. M. Spori, T. Drobek, S. Zürcher, and N. D. Spencer, "Cassie-State Wetting Investigated by Means of a Hole-to-Pillar Density Gradient," Langmuir, vol. 26, no. 12, pp. 9465-9473, 2010.

[24] F. J. Montes Ruiz-Cabello, and M. A. Rodríguez, "A new method for evaluating the most stable contact angle using tilting plate experiments," Soft Matter, vol. 7, no. 21, p. 10457, 2011.

[25] H. Mertaniemi, V. Jokinen, L. Sainiemi, S. Franssila, A. Marmur, O. Ikkala, and R. H. A. Ras, "Superhydrophobic Tracks for Low-Friction, Guided Transport of Water Droplets," Adv. Mater, vol. 23, no. 26, pp. 2911-2914, 2011. 